PROCEEDINGS OF THE

AMERICAN MATHEMATICAL SOCIETY

Volume 137, Number 6, June 2009, Pages 2037-2047

S 0002-9939(09)09805-0

Article electronically published on January 16, 2009

\title{
OPTIMAL REGULARITY FOR THE POISSON EQUATION
}

\author{
LIHE WANG, FENGPING YAO, SHULIN ZHOU, AND HUILIAN JIA
}

(Communicated by Matthew J. Gursky)

\begin{abstract}
In this paper we study the regularity theory for the Poisson equation in $\mathbb{R}^{n}$ under proper conditions. Furthermore, it will be verified that these conditions are optimal.
\end{abstract}

\section{INTRODUCTION}

Regularity theory in PDE plays an important role in the development of secondorder elliptic and parabolic equations. Classical regularity estimates for elliptic and parabolic equations consist of Schauder estimates, $L^{p}$ estimates, De GiorgiNash estimates, Krylov-Safanov estimates, and so on. When we study the $L^{p}$ estimates, it is necessary to introduce the notion of weak derivatives and to work in the so-called Sobolev spaces, which are sets of functions with a certain degree of smoothness. Sobolev spaces are commonly used and studied in a wide variety of fields of mathematics and have turned out to be one of the most powerful tools in analysis created in the 20th century.

Since the 1960s, the need to use wider spaces of functions than Sobolev spaces came from various practical problems. Orlicz spaces (see Definition 1.4) have been studied as the generalization of Sobolev spaces since they were introduced by Orlicz [11] (see [3, 5, 6, 7, 9, 10]). The theory of Orlicz spaces plays a crucial role in many fields of mathematics including geometric, probability, stochastic, Fourier analysis and partial differential equations (see 12]). In this paper we are concerned with the global regularity estimates in Orlicz spaces for the Poisson equation in $\mathbb{R}^{n}$ and the corresponding optimal conditions on $\phi$ (see (1.2)).

We consider the regularity for the following Poisson equation:

$$
-\Delta u=f \quad \text { in } \mathbb{R}^{n},
$$

where the dimension $n \geq 2$. Our main purpose in this work is to study what are the optimal conditions on those $\phi$ that satisfy an estimate such as

$$
\int_{\mathbb{R}^{n}} \phi\left(\left|D^{2} u\right|\right) d x \leq C \int_{\mathbb{R}^{n}} \phi(|f|) d x
$$

where $C$ is a positive constant independent of $u$ and $f$.

Received by the editors April 23, 2008, and, in revised form, July 21, 2008.

2000 Mathematics Subject Classification. Primary 35J05; Secondary 35J15.

Key words and phrases. Orlicz space, Poisson equation, regularity.

The first and fourth authors were supported in part by NSF \#0701392 and NSFC 10771166

The second and third authors were supported in part by the NBRPC under Grant 2006CB705700, the NSFC under Grant 60532080, and the KPCME under Grant 306017.

(c)2009 American Mathematical Society 
Indeed, if $\phi(t)=|t|^{p}$, (1.2) is reduced to the classical $L^{p}$ estimate (see [4, 8]). Moreover, Wang [13] gave a new proof of local $L^{p}$ estimates for the Poisson and heat equation by a geometric approach, in which the Hardy-Littlewood maximal function, modified Vitali covering lemma, and compactness method are used. Furthermore, using the same techniques as in [13, Jia, Li and Wang [9] generalized local estimates in $L^{p}$ space to Orlicz spaces for the Poisson equation when $\phi \in \Delta_{2} \cap \nabla_{2}$ (see Definitions 1.2 and 1.3). Since $\phi$ is not certain to be a polynomial, which leads to the failure of the normalization, the authors in 9 first assume that $u \in C_{0}^{\infty}(\Omega)$ and then use an interpolation inequality to obtain the result. Recently Acerbi and Mingione [1] obtained local $L^{q}, q \geq p$, gradient estimates for the degenerate parabolic $p$-Laplacian systems. There they invent a new iteration-covering approach, which is completely free from harmonic analysis. In this paper, encoding and simplifying the iteration-covering procedure used in [1] and extending it to the whole space (see Lemma 3.1 and Lemma 3.2), we will finish the proof of the estimate (1.2) under some assumptions on $\phi$ (see Theorem 1.5) and then verify that these assumptions are optimal.

Let $B_{r}=\left\{y \in \mathbb{R}^{n}:|y|<r\right\}$ be an open ball in $\mathbb{R}^{n}$ with center 0 and radius $r>0$, and $B_{r}(x)=B_{r}+x$. We denote

$$
\left|D^{2} u\right|=\sum_{i, j=1, \ldots, n}\left|D_{x_{i} x_{j}} u\right|
$$

and

$$
\left\|D^{2} u\right\|_{L^{p}\left(\mathbb{R}^{n}\right)}=\sum_{i, j=1, \ldots, n}\left\|D_{x_{i} x_{j}} u\right\|_{L^{p}\left(\mathbb{R}^{n}\right)}
$$

where

$$
\left\|D_{x_{i} x_{j}} u\right\|_{L^{p}\left(\mathbb{R}^{n}\right)}=\left(\int_{\mathbb{R}^{n}}\left|D_{x_{i} x_{j}} u\right|^{p} d x\right)^{1 / p} \text { for } p>1 .
$$

In this paper we denote by $\Phi$ the function class that consists of all functions $\phi:[0,+\infty) \longrightarrow[0,+\infty)$ which are increasing and convex.

Definition 1.1. A function $\phi \in \Phi$ is said to be a Young function if

$$
\lim _{t \rightarrow 0+} \frac{\phi(t)}{t}=\lim _{t \rightarrow+\infty} \frac{t}{\phi(t)}=0 .
$$

Definition 1.2. A Young function $\phi \in \Phi$ is said to satisfy the global $\nabla_{2}$ condition, denoted by $\phi \in \nabla_{2}$, if there exists a number $a>1$ such that for every $t>0$,

$$
\phi(t) \leq \frac{\phi(a t)}{2 a} .
$$

We can verify that if $\phi \in \nabla_{2}$, then $\phi$ satisfies for $0<\theta_{1} \leq 1$,

$$
\phi\left(\theta_{1} t\right) \leq 2 a \theta_{1}^{\alpha_{2}} \phi(t),
$$

where $\alpha_{2}=\log _{a} 2+1$.

Definition 1.3. A Young function $\phi \in \Phi$ is said to satisfy the global $\Delta_{2}$ condition, denoted by $\phi \in \Delta_{2}$, if there exists a positive constant $K$ such that for every $t>0$,

$$
\phi(2 t) \leq K \phi(t) .
$$


In fact, if $\phi \in \Delta_{2}$, then it is easy to check that $\phi$ satisfies for $1 \leq \theta_{2}<\infty$,

$$
\phi\left(\theta_{2} t\right) \leq K \theta_{2}^{\alpha_{1}} \phi(t),
$$

where $\alpha_{1}=\log _{2} K$. Moreover, we remark that the global $\Delta_{2} \cap \nabla_{2}$ condition makes the function grow moderately. For example, $\phi(t)=|t|^{\alpha}(1+|\log | t||)$ for $\alpha>1$ satisfies the global $\Delta_{2} \cap \nabla_{2}$ condition.

Definition 1.4. Let $\phi$ be a Young function. Then the Orlicz class $K^{\phi}\left(\mathbb{R}^{n}\right)$ is the set of all measurable functions $g: \mathbb{R}^{n} \rightarrow \mathbb{R}$ satisfying

$$
\int_{\mathbb{R}^{n}} \phi(|g|) d x<\infty
$$

The Orlicz space $L^{\phi}\left(\mathbb{R}^{n}\right)$ is the linear hull of $K^{\phi}\left(\mathbb{R}^{n}\right)$.

In general, $K^{\phi} \subset L^{\phi}$. However, if $\phi$ satisfies the global $\Delta_{2}$ condition, then $K^{\phi}=L^{\phi}$ and $C_{0}^{\infty}$ is dense in $L^{\phi}$ (see [2]). Moreover, we remark that if $g \in L^{\phi}\left(\mathbb{R}^{n}\right)$, then $\int_{\mathbb{R}^{n}} \phi(|g|) d x$ can be rewritten in an integral form as

$$
\int_{\mathbb{R}^{n}} \phi(|g|) d x=\int_{0}^{\infty}\left|\left\{x \in \mathbb{R}^{n}:|g|>\lambda\right\}\right| d[\phi(\lambda)] .
$$

Now let us state the main result of this work:

Theorem 1.5. Assume that $\phi \in \Phi$. If estimate (1.2) holds for every pair $(u, f) \in$ $C^{\infty}\left(\mathbb{R}^{n}\right) \times C_{0}^{\infty}\left(\mathbb{R}^{n}\right)$ satisfying equation (1.1) and $D^{2} u \in L^{\phi}\left(\mathbb{R}^{n}\right)$, then $\phi \in \Delta_{2} \cap \nabla_{2}$. On the contrary, if $\phi \in \Delta_{2} \cap \nabla_{2}$, then for every $f \in L^{\phi}\left(\mathbb{R}^{n}\right)$, there is a solution $u \in W_{\text {loc }}^{2,1}\left(\mathbb{R}^{n}\right)$ satisfying estimate (1.2).

Remark 1.6. Using the same approach, one can also deal with the parabolic case $u_{t}-\Delta u=f$ in $\mathbb{R}^{n} \times(0, T]$.

\section{Proof of the MAIN Result}

2.1. Necessity. In this subsection we show that $\phi \in \triangle_{2} \cap \nabla_{2}$ if estimate (1.2) is true.

Part 1. $\phi$ satisfies the global $\nabla_{2}$ condition.

Now we consider the special case in (1.1) when

$$
f_{t}(x)=t \eta
$$

where $t$ is a positive parameter, $\eta \in C_{0}^{\infty}\left(\mathbb{R}^{n}\right)$ is a cutoff function satisfying

$$
0 \leq \eta(x) \leq 1, \quad \eta(x) \equiv 1 \quad \text { in } B_{1}, \quad \eta(x)=0 \text { in } \mathbb{R}^{n} / B_{2} .
$$

Therefore equation (1.1) has a solution

$$
u_{t}(x)=\int_{\mathbb{R}^{n}} \Gamma(x-\xi) f_{t}(\xi) d \xi,
$$

where

$$
\Gamma(x)= \begin{cases}\frac{1}{n(n-2) w_{n}} \frac{1}{|x|^{n-2}} & (n>2), \\ -\frac{1}{2 \pi} \ln |x| & (n=2)\end{cases}
$$

is the fundamental solution of $-\triangle$. 
It follows from (1.2) and (2.1) that

$$
\int_{\mathbb{R}^{n}} \phi\left(\left|D^{2} u\right|\right) d x \leq C \int_{\mathbb{R}^{n}} \phi\left(\left|f_{t}\right|\right) d x \leq C \phi(t) .
$$

We know from (2.2) that

$$
D_{x_{i} x_{i}} u_{t}(x)=\int_{B_{2}} \frac{1}{w_{n}|x-\xi|^{n}}\left[\frac{\left(x_{i}-\xi_{i}\right)^{2}}{|x-\xi|^{2}}-\frac{1}{n}\right] f_{t}(\xi) d \xi
$$

when $|x|>2$.

We define

$$
D=:\left\{x \in \mathbb{R}^{n}:|x|>12 \sqrt{n} \text { and }\left|x_{1}\right| \geq \frac{4}{3} \frac{1}{\sqrt{n}}|x|\right\} .
$$

When $x \in D, \xi \in B_{2} \backslash B_{1}$, we have

$$
\frac{\left|x_{1}-\xi_{1}\right|}{|x-\xi|} \geq \frac{\left|x_{1}\right|-2}{|x|+2} \geq \frac{\frac{4}{3} \frac{1}{\sqrt{n}}|x|-2}{|x|+2} \geq \frac{1}{\sqrt{n}} .
$$

When $x \in D, \xi \in B_{1}$, we have

$$
\frac{\left|x_{1}-\xi_{1}\right|}{|x-\xi|} \geq \frac{\left|x_{1}\right|-1}{|x|+1} \geq \frac{\frac{4}{3} \frac{1}{\sqrt{n}}|x|-1}{|x|+1} \geq \frac{7}{6} \frac{1}{\sqrt{n}}
$$

and

$$
|x-\xi| \leq|x|+|\xi| \leq|x|+1 \leq \frac{3}{2}|x| .
$$

Therefore, for $x \in D$ we conclude that

$$
\begin{aligned}
D_{x_{1} x_{1}} u_{t}(x) & =t \int_{B_{2}} \frac{1}{w_{n}|x-\xi|^{n}}\left[\frac{\left(x_{1}-\xi_{1}\right)^{2}}{|x-\xi|^{2}}-\frac{1}{n}\right] \eta d \xi \\
& \geq t \frac{2^{n}}{3^{n}} \frac{1}{w_{n}|x|^{n}} \int_{B_{1}}\left[\left(\frac{7}{6}\right)^{2} \frac{1}{n}-\frac{1}{n}\right] d \xi \\
& \geq \frac{t}{3^{n} n^{2}}|x|^{-n} .
\end{aligned}
$$

Recalling estimate (2.3) we find that

$$
\int_{D} \phi\left(\frac{t}{3^{n} n^{2}}|x|^{-n}\right) d x \leq C \phi(t)
$$

which implies that

$$
\int_{12 \sqrt{n}}^{+\infty} \phi\left(\frac{t}{3^{n} n^{2}} r^{-n}\right) r^{n-1} d r \int_{\left|\cos \theta_{1}\right|>\frac{4}{3} \frac{1}{\sqrt{n}}} d \omega \leq C \phi(t) .
$$

By changing the variable we conclude that, for $t>0$,

$$
\int_{0}^{\alpha t} \frac{\phi(s)}{s^{2}} d s \leq \frac{C \phi(t)}{t},
$$

where $\alpha=48^{-n} n^{-2-\frac{n}{2}}$. 
Let $t_{2} \geq t_{1}, 0<\varepsilon \leq \frac{\alpha}{2}$. Then we conclude from (2.5) that

$$
\begin{aligned}
\frac{\phi\left(t_{2}\right)}{t_{2}} & \geq \frac{1}{C} \int_{0}^{\alpha t_{2}} \frac{\phi(s)}{s^{2}} d s \geq \frac{1}{C} \int_{\varepsilon t_{1}}^{\alpha t_{1}} \frac{\phi(s)}{s^{2}} d s \\
& \geq \frac{\phi\left(\varepsilon t_{1}\right)}{C}\left(\frac{1}{\varepsilon t_{1}}-\frac{1}{\alpha t_{1}}\right) \geq \frac{\phi\left(\varepsilon t_{1}\right)}{2 C \varepsilon t_{1}} .
\end{aligned}
$$

Now we use (2.5) and (2.6) to obtain that

$$
\frac{\phi(t)}{t} \geq \frac{1}{C} \int_{\varepsilon t}^{\alpha t} \frac{\phi(s)}{s} \frac{1}{s} d s \geq \frac{\phi\left(\varepsilon^{2} t\right)}{2 C^{2} \varepsilon^{2} t} \ln \frac{\alpha}{\varepsilon},
$$

where we choose that $t_{1}=\varepsilon t, t_{2}=s$ in (2.6).

Set $a=1 / \varepsilon^{2}$. Then we have

$$
\phi(t) \geq \frac{\ln (\alpha \sqrt{a})}{2 C^{2}} a \phi\left(\frac{t}{a}\right) \geq 2 a \phi\left(\frac{t}{a}\right),
$$

when $a$ is chosen large enough. This implies that $\phi$ satisfies the $\nabla_{2}$ condition.

Part 2. $\phi$ satisfies the global $\Delta_{2}$ condition.

Define two constants,

$$
C_{1}=\max _{x \in \mathbb{R}^{n}}\left\{|\Delta \eta|=\left|\sum_{i=1}^{n} D_{x_{i} x_{i}} \eta\right|\right\}
$$

and

$$
C_{2}=\max _{x \in \mathbb{R}^{n}}\left\{\left|D^{2} \eta\right|=\sum_{i, j=1, \ldots, n}\left|D_{x_{i} x_{j}} \eta\right|\right\},
$$

where $\eta(x) \in C_{0}^{\infty}\left(\mathbb{R}^{n}\right)$ is a cutoff function defined in (2.1). It is easy to see that $C_{2}>C_{1}$. Now we consider the special case in (1.1) when

$$
u_{t}(x)=\frac{t \eta(x)}{C_{1}} \text { and } f_{t}(x)=-\frac{t \triangle \eta(x)}{C_{1}},
$$

where $t$ is a positive parameter.

From the proof of Part 1, we know that $\phi \in \nabla_{2}$ if estimate (1.2) is true. Set

$$
C_{3}=\frac{C_{1}+C_{2}}{2}, \quad \gamma=\frac{C_{3}}{C_{1}} .
$$

It is obvious that $\gamma>1$. Then from (1.2) and (1.3) we obtain

$$
\begin{aligned}
\phi(\gamma t)\left|\left\{x \in \mathbb{R}^{n}:\left|D^{2} \eta\right|>C_{3}\right\}\right| & =\phi(\gamma t)\left|\left\{x \in \mathbb{R}^{n}:\left|D^{2} u_{t}\right|>\gamma t\right\}\right| \\
& \leq \int_{\mathbb{R}^{n}} \phi\left(\left|D^{2} u_{t}\right|\right) d x \\
& \leq C \int_{\mathbb{R}^{n}} \phi\left(\left|f_{t}\right|\right) d x \\
& \leq C \phi(t) \int_{\mathbb{R}^{n}} 2 a\left(\frac{|\Delta \eta|}{C_{1}}\right)^{\alpha_{2}} d x \\
& \leq C \phi(t) .
\end{aligned}
$$

Therefore, we conclude that

$$
\phi(\gamma t) \leq C \phi(t)
$$


which implies that

$$
\phi(2 t) \leq C \phi(t)
$$

This completes our proof.

\section{SUfFICIENCY}

In Subsection 3.1 we use an approximation argument to show that the proof of the sufficiency part of Theorem 1.5 can be reduced to proving an a priori estimate (1.2) with the assumption that $D^{2} u \in L^{\phi}\left(\mathbb{R}^{n}\right)$, where $\phi$ satisfies both the $\triangle_{2}$ and the $\nabla_{2}$ conditions. In Subsection 3.2, we shall finish the proof of the sufficiency part of Theorem 1.5 .

3.1. Approximation. Let $\left\{f_{k}\right\}_{k=1}^{\infty}$ be a sequence of smooth functions in $C_{0}^{\infty}\left(\mathbb{R}^{n}\right)$ satisfying

$$
f_{k} \longrightarrow f \text { in } L^{\phi}\left(\mathbb{R}^{n}\right)
$$

for a given Young function $\phi \in \triangle_{2} \cap \nabla_{2}$. Then

$$
\int_{\mathbb{R}^{n}} \phi\left(\left|f_{k}\right|\right) d x \longrightarrow \int_{\mathbb{R}^{n}} \phi(|f|) d x
$$

Now we consider the regularized problems

$$
-\Delta u_{k}=f_{k} \in C_{0}^{\infty}\left(\mathbb{R}^{n}\right) \text { in } \mathbb{R}^{n} .
$$

Then we have

$$
\int_{\mathbb{R}^{n}}\left|D^{2} u_{k}\right|^{\alpha_{1}} d x \leq C \int_{\mathbb{R}^{n}}\left|f_{k}\right|^{\alpha_{1}} d x, \int_{\mathbb{R}^{n}}\left|D^{2} u_{k}\right|^{\alpha_{2}} d x \leq C \int_{\mathbb{R}^{n}}\left|f_{k}\right|^{\alpha_{2}} d x,
$$

where $\alpha_{1}$ and $\alpha_{2}$ are defined in (1.3) and (1.4). Therefore, it follows from the above inequality, (1.3) and (1.4) that

$$
\begin{aligned}
\int_{\mathbb{R}^{n}} \phi\left(\left|D^{2} u_{k}\right|\right) d x & =\int_{\left|D^{2} u_{k}\right| \geq 1} \phi\left(\left|D^{2} u_{k}\right|\right) d x+\int_{\left|D^{2} u_{k}\right|<1} \phi\left(\left|D^{2} u_{k}\right|\right) d x \\
& \leq K \phi(1) \int_{\mathbb{R}^{n}}\left|D^{2} u_{k}\right|^{\alpha_{1}} d x+2 a \phi(1) \int_{\mathbb{R}^{n}}\left|D^{2} u_{k}\right|^{\alpha_{2}} d x \\
& \leq C\left(\int_{\mathbb{R}^{n}}\left|f_{k}\right|^{\alpha_{1}} d x+\int_{\mathbb{R}^{n}}\left|f_{k}\right|^{\alpha_{2}} d x\right) \leq C .
\end{aligned}
$$

Thus our estimate in Subsection 3.2 implies that

$$
\int_{\mathbb{R}^{n}} \phi\left(\left|D^{2} u_{k}\right|\right) d x \leq C \int_{\mathbb{R}^{n}} \phi\left(\left|f_{k}\right|\right) d x,
$$

where the constant $C$ is independent of $k \in \mathbb{N}$. Finally, letting $k \rightarrow \infty$ and using (3.1) and the lower semicontinuity to handle the left-hand side of (3.2), we obtain the a priori estimate (1.2); that is,

$$
\int_{\mathbb{R}^{n}} \phi\left(\left|D^{2} u\right|\right) d x \leq C \int_{\mathbb{R}^{n}} \phi(|f|) d x .
$$


3.2. Final proof. We need two important lemmas, which are much motivated by Step 2 and Step 4 (i.e., the iteration-covering procedure) in [1. Let

$$
p=\left(1+\alpha_{2}\right) / 2>1 .
$$

In fact, in the subsequent proof we can choose any constant $p$ with $1<p<\alpha_{2}$. Now we write

$$
\lambda_{0}^{p}=\int_{\mathbb{R}^{n}}\left|D^{2} u\right|^{p} d x+M^{p} \int_{\mathbb{R}^{n}}|f|^{p} d x,
$$

while $M>1$ is a large enough constant which will be determined later. Set

$$
u_{\lambda}=u /\left(\lambda_{0} \lambda\right) \text { and } f_{\lambda}=f /\left(\lambda_{0} \lambda\right)
$$

for any $\lambda>0$. Then $u_{\lambda}$ is still the solution of (1.1) with $f_{\lambda}$ replacing $f$. Moreover, for any domain $B$ in $\mathbb{R}^{n}$ we write

$$
J_{\lambda}[B]=f_{B}\left|D^{2} u_{\lambda}\right|^{p} d x+M^{p} f_{B}\left|f_{\lambda}\right|^{p} d x
$$

and

$$
E_{\lambda}(1)=\left\{x \in \mathbb{R}^{n}:\left|D^{2} u_{\lambda}\right|>1\right\} .
$$

Since $\left|D^{2} u_{\lambda}(x)\right| \leq 1$ for $x \in \mathbb{R}^{n} \backslash E_{\lambda}(1)$, we focus our attention on the level set $E_{\lambda}(1)$. Next, we will decompose the level set $E_{\lambda}(1)$.

Lemma 3.1. For any $\lambda>0$, there exists a family of disjoint balls $\left\{B_{\rho_{i}}\left(x_{i}\right)\right\}_{i \geq 1}$ with $x_{i} \in E_{\lambda}(1)$ and $\rho_{i}=\rho\left(x_{i}, \lambda\right)>0$ such that

$$
J_{\lambda}\left[B_{\rho_{i}}\left(x_{i}\right)\right]=1, \quad J_{\lambda}\left[B_{\rho}\left(x_{i}\right)\right]<1 \text { for any } \rho>\rho_{i},
$$

and

$$
E_{\lambda}(1) \subset \bigcup_{i \geq 1} B_{5 \rho_{i}}\left(x_{i}\right) \cup \text { negligible set. }
$$

Proof. Fix any $x \in \mathbb{R}^{n}$ and $\rho \geq \rho_{0}=\rho_{0}(\lambda)>0$ with $\lambda^{p}\left|B_{\rho_{0}}\right|=1$. Then it follows from (3.4) that

$$
J_{\lambda}\left[B_{\rho}(x)\right] \leq \frac{1}{\left|B_{\rho}(x)\right|}\left[\int_{\mathbb{R}^{n}}\left|D^{2} u_{\lambda}\right|^{p} d x+M^{p} \int_{\mathbb{R}^{n}}\left|f_{\lambda}\right|^{p} d x\right] \leq \frac{1}{\left|B_{\rho}(x)\right| \lambda^{p}} \leq 1 .
$$

Thus we conclude that

$$
\sup _{x \in \mathbb{R}^{n}} \sup _{\rho \geq \rho_{0}} J_{\lambda}\left[B_{\rho}(x)\right] \leq 1 .
$$

For a.e. $x \in E_{\lambda}(1)$, by Lebesgue's differentiation theorem we know that

$$
\lim _{\rho \rightarrow 0} J_{\lambda}\left[B_{\rho}(x)\right]>1,
$$

which implies that there exists some $\rho>0$ satisfying

$$
J_{\lambda}\left[B_{\rho}(x)\right]>1 .
$$

Therefore from (3.7) one can select $\rho_{x} \in\left(0, \rho_{0}\right]$ such that

$$
J_{\lambda}\left[B_{\rho_{x}}(x)\right]=1, \quad J_{\lambda}\left[B_{\rho}(x)\right]<1 \text { for any } \rho>\rho_{x} .
$$

It follows from the argument above that for a.e. $x \in E_{\lambda}(1)$ there exists a ball $B_{\rho_{x}}(x)$ constructed as above. Therefore, applying Vitali's covering lemma, we can find a family of disjoint balls $\left\{B_{\rho_{i}}\left(x_{i}\right)\right\}_{i \geq 1}$ such that the results of the lemma hold. This completes our proof. 
Next, we obtain the following estimates of the balls $\left\{B_{\rho_{i}}\left(x_{i}\right)\right\}$.

Lemma 3.2. Under the same hypotheses and results as those in Lemma 3.1, we have

$$
\begin{aligned}
\left|B_{\rho_{i}}\left(x_{i}\right)\right| \leq \frac{2^{p-1}}{2^{p-1}-1}( & \int_{\left\{x \in B_{\rho_{i}}\left(x_{i}\right):\left|D^{2} u_{\lambda}\right|>1 / 2\right\}}\left|D^{2} u_{\lambda}\right|^{p} d x \\
& \left.+M^{p} \int_{\left\{x \in B_{\rho_{i}}\left(x_{i}\right):\left|f_{\lambda}\right|>1 /(2 M)\right\}}\left|f_{\lambda}\right|^{p} d x\right) .
\end{aligned}
$$

Proof. From (3.6) in the lemma above we see that

$$
\left|B_{\rho_{i}}\left(x_{i}\right)\right|=\int_{B_{\rho_{i}}\left(x_{i}\right)}\left|D^{2} u_{\lambda}\right|^{p} d x+M^{p} \int_{B_{\rho_{i}}\left(x_{i}\right)}\left|f_{\lambda}\right|^{p} d x .
$$

Therefore, by splitting the two integrals above as follows we have

$$
\begin{aligned}
\left|B_{\rho_{i}}\left(x_{i}\right)\right| \leq & \int_{\left\{x \in B_{\rho_{i}}\left(x_{i}\right):\left|D^{2} u_{\lambda}\right|>1 / 2\right\}}\left|D^{2} u_{\lambda}\right|^{p} d x+(1 / 2)^{p}\left|B_{\rho_{i}}\left(x_{i}\right)\right| \\
& +M^{p} \int_{\left\{x \in B_{\rho_{i}}\left(x_{i}\right):\left|f_{\lambda}\right|>1 /(2 M)\right\}}\left|f_{\lambda}\right|^{p} d x+(1 / 2)^{p}\left|B_{\rho_{i}}\left(x_{i}\right)\right| .
\end{aligned}
$$

Thus we have concluded with the desired estimate.

For the proof of sufficiency in Theorem 1.5, we need the following result.

Lemma 3.3. If $\phi \in \Phi$ satisfies the global $\triangle_{2} \cap \nabla_{2}$ condition, then for any $b_{1}, b_{2}>0$ we have

$$
\int_{0}^{\infty} \frac{1}{\mu^{p}}\left\{\int_{\left\{x \in \mathbb{R}^{n}:|g|>b_{1} \mu\right\}}|g|^{p} d x\right\} d\left[\phi\left(b_{2} \mu\right)\right] \leq C\left(b_{1}, b_{2}, \phi\right) \int_{\mathbb{R}^{n}} \phi(|g|) d x .
$$

Proof. Interchanging the order of integration and integrating by parts, we deduce that

$$
\begin{aligned}
I & =: \int_{\mathbb{R}^{n}}|g|^{p}\left\{\int_{0}^{|g| / b_{1}} \frac{d\left[\phi\left(b_{2} \mu\right)\right]}{\mu^{p}}\right\} d x \\
& \leq \int_{\mathbb{R}^{n}}|g|^{p}\left\{\frac{\phi\left(b_{2}|g| / b_{1}\right)}{\left(|g| / b_{1}\right)^{p}}+p \int_{0}^{|g| / b_{1}} \frac{\phi\left(b_{2} \mu\right)}{\mu^{p+1}} d \mu\right\} d x,
\end{aligned}
$$

and it follows from (1.3), (1.4) and (3.3) that

$$
\begin{aligned}
I & \leq C \int_{\mathbb{R}^{n}} \phi(|g|) d x+2 a p b_{1}^{\alpha_{2}} \int_{\mathbb{R}^{n}} \phi\left(b_{2}|g| / b_{1}\right)|g|^{p-\alpha_{2}}\left\{\int_{0}^{|g| / b_{1}} \frac{1}{\mu^{p+1-\alpha_{2}}} d \mu\right\} d x \\
& \leq C \int_{\mathbb{R}^{n}} \phi(|g|) d x .
\end{aligned}
$$

Thus we complete the proof.

Now we are set to prove the sufficiency in Theorem 1.5 .

Proof. Fix $i \geq 1$. In view of Lemma 3.1, we obtain

$$
f_{B_{10 \rho_{i}}\left(x_{i}\right)}\left|D^{2} u_{\lambda}\right|^{p} d x \leq 1 \text { and } f_{B_{10 \rho_{i}}\left(x_{i}\right)}\left|f_{\lambda}\right|^{p} d x \leq \frac{1}{M^{p}} .
$$


Now let $v$ be a solution of

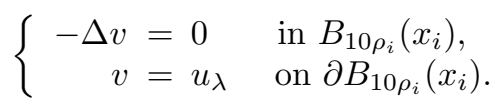

Set $w=u_{\lambda}-v$. Then $w$ satisfies $-\Delta w=f_{\lambda}$ in $B_{10 \rho_{i}}\left(x_{i}\right)$ with the boundary condition $w=0$ on $\partial B_{10 \rho_{i}}\left(x_{i}\right)$. Thus from the elementary $L^{p}$ estimates and (3.8) we find that

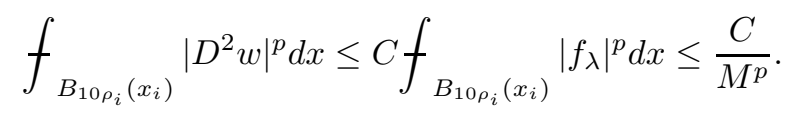

Furthermore, we conclude from (3.9) that

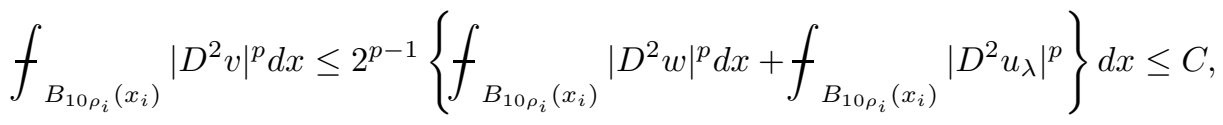

from which it follows using $W_{l o c}^{2, \infty}$ regularity that

$$
\sup _{B_{5 \rho_{i}}\left(x_{i}\right)}\left|D^{2} v\right| \leq N_{1}
$$

where $N_{1}>1$ only depends on $n, p$. Set $\mu=\lambda \lambda_{0}$. Then we deduce from (3.5), (3.9) and (3.10) that

$$
\begin{aligned}
& \left|\left\{x \in B_{5 \rho_{i}}\left(x_{i}\right):\left|D^{2} u\right|>2 N_{1} \mu\right\}\right|=\left|\left\{x \in B_{5 \rho_{i}}\left(x_{i}\right):\left|D^{2} u_{\lambda}\right|>2 N_{1}\right\}\right| \\
& \leq\left|\left\{x \in B_{5 \rho_{i}}\left(x_{i}\right):\left|D^{2} w\right|>N_{1}\right\}\right|+\left|\left\{x \in B_{5 \rho_{i}}\left(x_{i}\right):\left|D^{2} v\right|>N_{1}\right\}\right| \\
& =\left|\left\{x \in B_{5 \rho_{i}}\left(x_{i}\right):\left|D^{2} w\right|>N_{1}\right\}\right| \leq \frac{1}{N_{1}^{p}} \int_{B_{5 \rho_{i}}\left(x_{i}\right)}\left|D^{2} w\right|^{p} d x \leq \frac{C\left|B_{\rho_{i}}\left(x_{i}\right)\right|}{M^{p}} .
\end{aligned}
$$

It then follows from Lemma 3.2 and (3.5) that

$$
\begin{aligned}
& \left|\left\{x \in B_{5 \rho_{i}}\left(x_{i}\right):\left|D^{2} u\right|>2 N_{1} \mu\right\}\right| \\
& \leq \frac{C_{1}}{M^{p} \mu^{p}}\left(\int_{\left\{x \in B_{\rho_{i}}\left(x_{i}\right):\left|D^{2} u\right|>\mu / 2\right\}}\left|D^{2} u\right|^{p} d x+M^{p} \int_{\left\{x \in B_{\rho_{i}}\left(x_{i}\right):|f|>\mu /(2 M)\right\}}|f|^{p} d x\right),
\end{aligned}
$$

where $C_{1}=C_{1}(n, \phi)$. Then recalling the fact that the balls $\left\{B_{\rho_{i}}\left(x_{i}\right)\right\}$ are disjoint and

$$
\bigcup_{i \geq 1} B_{5 \rho_{i}}\left(x_{i}\right) \cup \text { negligible set } \supset E_{\lambda}(1)=\left\{x \in \mathbb{R}^{n}:\left|D^{2} u_{\lambda}\right|>1\right\}
$$

for any $\lambda>0$, we have

$$
\begin{aligned}
& \left|\left\{x \in \mathbb{R}^{n}:\left|D^{2} u\right|>2 N_{1} \mu\right\}\right| \leq \sum_{i}\left|\left\{x \in B_{5 \rho_{i}}\left(x_{i}\right):\left|D^{2} u\right|>2 N_{1} \mu\right\}\right| \\
& \leq \frac{C_{1}}{M^{p} \mu^{p}}\left(\int_{\left\{x \in \mathbb{R}^{n}:\left|D^{2} u\right|>\mu / 2\right\}}\left|D^{2} u\right|^{p} d x+M^{p} \int_{\left\{x \in \mathbb{R}^{n}:|f|>\mu /(2 M)\right\}}|f|^{p} d x\right) .
\end{aligned}
$$


Furthermore, recalling (1.5) and Lemma 3.3. we compute

$$
\begin{aligned}
\int_{\mathbb{R}^{n}} \phi\left(\left|D^{2} u\right|\right) d x= & \int_{0}^{\infty}\left|\left\{x \in \mathbb{R}^{n}:\left|D^{2} u\right|>2 N_{1} \mu\right\}\right| d\left[\phi\left(2 N_{1} \mu\right)\right] \\
\leq & \frac{C_{1}}{M^{p}} \int_{0}^{\infty} \frac{1}{\mu^{p}}\left\{\int_{\left\{x \in \mathbb{R}^{n}:\left|D^{2} u\right|>\mu / 2\right\}}\left|D^{2} u\right|^{p} d x\right\} d\left[\phi\left(2 N_{1} \mu\right)\right] \\
& +C_{1} \int_{0}^{\infty} \frac{1}{\mu^{p}}\left\{\int_{\left\{x \in \mathbb{R}^{n}:|f|>\mu /(2 M)\right\}}|f|^{p} d x\right\} d\left[\phi\left(2 N_{1} \mu\right)\right] \\
\leq & \frac{C_{2}}{M^{p}} \int_{\mathbb{R}^{n}} \phi\left(\left|D^{2} u\right|\right) d x+C_{3} \int_{\mathbb{R}^{n}} \phi(|f|) d x,
\end{aligned}
$$

where $C_{2}=C_{2}(n, \phi)$ and $C_{3}=C_{3}(n, \phi, M)$. Finally, choosing a suitable $M>0$ such that $C_{2} / M^{p}<1 / 2$, we obtain

$$
\int_{\mathbb{R}^{n}} \phi\left(\left|D^{2} u\right|\right) d x \leq C \int_{\mathbb{R}^{n}} \phi(|f|) d x .
$$

Thus we complete the proof.

\section{ACKNOWLEDGMENT}

The authors wish to thank the anonymous referee for offering valuable suggestions to improve the expressions.

\section{REFERENCES}

[1] E. Acerbi and G. Mingione, Gradient estimates for a class of parabolic systems, Duke Math. J., 136 (2007), 285-320. MR2286632 (2007k:35211)

[2] R. A. Adams and J. J. F. Fournier, Sobolev spaces (2nd edition), Elsevier/Academic Press, Amsterdam-New York, 2003. MR2424078

[3] A. Benkirane and A. Elmahi, An existence theorem for a strongly nonlinear elliptic problem in Orlicz spaces, Nonlinear Anal., 36 (1999), 11-24. MR.1670307(99m:35056)

[4] Y. Chen and L. Wu, Second order elliptic equations and elliptic systems, American Mathematical Society, Providence, RI, 1998. MR,1616087 (99i:35016)

[5] A. Cianchi, Hardy inequalities in Orlicz spaces, Trans. Amer. Math. Soc., 351 (1999), 24592478. MR1433113 (99i:46016)

[6] T. Donaldson, Nonlinear elliptic boundary value problems in Orlicz-Sobolev spaces, J. Diff. Eq., 10 (1971), 507-528. MR0298472 (45:7524)

[7] T. Donaldson, Inhomogeneous Orlicz-Sobolev spaces and nonlinear parabolic initial value problems, J. Diff. Eq., 16 (1974), 201-256. MR0380384 (52:1284)

[8] D. Gilbarg and N. Trudinger, Elliptic partial differential equations of second order (reprint of the 1998 edition), Springer-Verlag, Berlin, 2001. MR.1814364(2001k:35004)

[9] H. Jia, D. Li and L. Wang, Regularity of Orlicz spaces for the Poisson equation, Manuscripta Mathematica, 122 (2007), 265-275. MR2305417 (2007m:35036)

[10] V. Kokilashvili and M. Krbec, Weighted inequalities in Lorentz and Orlicz spaces, World Scientific Publishing Co., River Edge, NJ, 1991. MR1156767 (93g:42013)

[11] W. Orlicz, Üeber eine gewisse Klasse von Räumen vom Typus B, Bull. Int. Acad. Pol. Ser. A, 8 (1932), 207-220.

[12] M. Rao and Z. Ren, Applications of Orlicz spaces, Marcel Dekker, New York, 2002. MR 1890178 (2003e:46041)

[13] L. Wang, A geometric approach to the Calderón-Zygmund estimates. Acta Math. Sin. (Engl. Ser.), 19 (2) (2003), 381-396. MR 1987802 (2004e:42033) 
Department of Mathematics, University of Iowa, Iowa City, Iowa 52242

Current address: Department of Mathematics, Xian Jiaotong University, Xian 710049, People's Republic of China

E-mail address: lwang@math.uiowa.edu

Department of Mathematics, Shanghai University, Shanghai 200444, People's RepubLIC OF CHINA

E-mail address: yfp@shu.edu.cn

LMam, School of Mathematical Sciences, Peking University, Beijing 100871, PeoPLE'S REPUBlic of CHINA

E-mail address: szhou@math.pku.edu.cn

Department of Mathematics, Xian Jiaotong University, Xian 710049, People's RepubLIC OF CHINA

E-mail address: jiahl@mail.xjtu.edu.cn 